\title{
Fuentes de información documentales filosófico-jurídicas
}

\author{
Isabel VILLASEÑOR RODRÍGUEZ \\ Universidad Complutense de Madrid \\ isavilla@pdi.ucm.es
}

Recibido: 19/06/2011

Aceptado: 04/07/2011

\section{RESUMEN}

El presente artículo ofrece una aproximación a las que han sido, y siguen siendo, las fuentes de información documentales de mayor utilidad para los filósofos del Derecho en España, fundamentalmente desde el siglo XIX hasta nuestros días. Se analiza el desarrollo experimentado por algunas de ellas y se presentan algunos ejemplos de interés. Las conclusiones recogen información original respecto a este tipo de Documentación.

Palabras clave: Fuentes de información documentales. Documentación filosófico-jurídica. Fuentes de información especializadas. Documentación especializada.

\section{Documentary sources of information on legal philosophy}

\begin{abstract}
This article offers an approach to which they have been, and remain, the documentary sources of information more useful to the philosophical and legal documentation in Spain, mainly from the nineteenth century to today. It analyzes the development experienced by some of them, with some examples of interest. The conclusions reflect the original information about this type of documentation.
\end{abstract}

Key words: Documentary sources of information. Philosophical and legal documentation. Specialized information sources. Specialized documentation.

\section{INTRODUCCIÓN}

La Filosofía jurídica es la disciplina que proporciona la perspectiva filosófica en torno al Derecho, y, por tanto, más abierta y amplia que la perspectiva dogmáticojurídica, la cual es propia de la mayor parte de ramas de la Ciencia jurídica. Esta disciplina genera y consume una serie de fuentes de información que, consideradas en su conjunto, constituyen lo que se ha dado en llamar Documentación filosófico-jurídica. Se trata de una Documentación que posee rasgos que la hacen particular con respecto a la más genérica categoría con la que se relaciona, conocida con el nombre de 
Documentación jurídica. En esta relación, la Documentación filosófico-jurídica posee las características propias de la que algunos autores ${ }^{1}$ han denominado Documentación de la doctrina jurídica o Bibliografia jurídica, entendida como cualquier estudio sobre temas jurídicos. Esta Documentación, a diferencia de otras categorías propias de la Documentación jurídica, como son la Documentación legislativa y la judicial, se caracteriza por ser una Documentación poco controlada, sin tipificación ni estructura definida y con una vigencia generalmente ilimitada. Se trata de una Documentación que utiliza un lenguaje de léxico especial y propio del tipo de información que transmite. Por lo general, sigue una difusión diferente al resto (repertorios de legislación y jurisprudencia, publicaciones oficiales...) a través de editoriales y revistas especializadas. Su publicidad y referencia se logra por medio de catálogos oficiales y privados. Integrada fundamentalmente por los tradicionales documentos bibliográficos en los que se publica la literatura jurídica, independientemente del soporte o del formato, como son las monografías, los manuales, los artículos de publicaciones periódicas, las actas de congresos y conferencias y la "literatura gris", goza de un alto nivel de publicación. Se presta, mejor que las otras, al resumen en su tratamiento documental. Aunque la edición impresa juega un papel preponderante, las ediciones electrónicas (libros y revistas) se están integrando paulatinamente en la oferta de las principales editoriales jurídicas. Las bases de datos bibliográficas de carácter jurídico, por su concepción y finalidad se asemejan al resto de bases de datos referenciales sin que quepa hacer diferenciación alguna en su forma de almacenamiento y utilización práctica. Reciben el nombre de bases de datos doctrinales y recogen, de forma referencial, la doctrina jurídica publicada en libros, revistas, artículos de prensa, "literatura gris", etc. Páez Mañá dice que la Documentación de la doctrina jurídica está constituida por documentos que contienen mensajes jurídicos (ideas, hechos, opiniones) y que están avalados "por el prestigio jurídico alcanzado por sus autores siendo fuente documental del conocimiento del Derecho, teniendo como finalidad añadida la de sensibilizar la conciencia social"2. En muchos casos se centran en el análisis de los textos legales y jurisprudenciales.

De esta manera podemos afirmar que la Documentación filosófico-jurídica ${ }^{3}$ es, teniendo en cuenta lo dicho hasta el momento, Documentación de la doctrina jurídica; esto es, Documentación referida a todo aquello que atañe a la vastísima problemática que implica la consideración del Derecho como un producto cultural en el sentido más amplio del término.

${ }^{1}$ Autores como MACIÁ y REVIRIEGO (MANUAL de documentación jurídica. Edit. por Mateo Maciá. Madrid: Síntesis, 1998) y PÁEZ MAÑÁ (Bases de datos jurídicos. Madrid: CINDOC, CSIC, 1994) la llaman "doctrina científica".

2 Op. cit., p. 5.

${ }^{3}$ Utilizaremos como sinónimos los términos "filosófico-jurídica"/“iusfilosófica" para referirnos a la Documentación objeto de nuestro interés porque así se acepta entre los usuarios y autores de este tipo de Documentación. 
En cuanto a las fuentes de información ${ }^{4}$ propias de esta Documentación, que mayor interés y auge han tenido y tienen entre los usuarios de esta disciplina han sido y son las fuentes documentales de carácter primario, es decir, aquellas fuentes destinadas a transmitir los resultados del conocimiento y de la creación en distintos soportes. $\mathrm{Su}$ desarrollo se da fundamentalmente a partir del siglo XIX y, sobre todo, desde el XX, como ocurre con otros tipos de fuentes de información documentales, como son las que remiten a otras fuentes o reelaboran la información contenida en ellas (las secundarias), $\mathrm{y}$ las fuentes documentales terciarias.

\section{EL CONTROL DE LAS FUENTES DE INFORMACIÓN DOCUMENTALES}

La principal dificultad que se nos plantea es la ausencia de recursos de control que den cuenta de fuentes de información documentales de interés. Quizá podríamos considerar los manuales creados por profesores de una asignatura (Bibliografia jurídica) que se impartió en la Facultad de Derecho de la Universidad Central ${ }^{6}$ como "guías de fuentes", pero su rastreo no nos proporciona la suficiente información, ya que, aunque especializados en Derecho, apenas ofrecen referencias de fuentes de información iusfilosófica. En cuanto a las fuentes documentales, el control bibliográfico en nuestro país ha significado un verdadero problema a pesar de las distintas tentativas que han existido, tanto privadas como públicas. Entre las primeras cabe destacar la llevada a cabo por libreros, sobre todo de antiguo que, como asegura Botrel $^{7}$, se preocuparon del control bibliográfico editorial español en iniciativas individuales que no tuvieron la continuidad, la seriedad ni la exhaustividad deseadas. Hubo algunos intentos de control bibliográfico con publicaciones a cargo de asociaciones como la de la Librería Española ("Bibliografía española", por ejemplo). En cuanto a la iniciativa pública contamos con actuaciones tales como la creación del Depósito Legal (en 1716) o el desarrollo de la bibliografía nacional de carácter oficial (ya en el siglo XX, en 1958), que permite conocer el "paisaje" intelectual de un país por ser testigo de la memoria nacional ${ }^{8}$. A lo largo de los siglos, la producción aumenta, no sólo en número sino también en variedad. La eclosión bibliográfica experimentada hacia la segunda

${ }^{4}$ Un desarrollo de este tema se encuentra en VILLASEÑOR RODRÍGUEZ, Isabel: Fuentes de información en filosofía jurídica española (siglos XIX-XXI). Madrid: Dykinson, 2009.

${ }^{5}$ Para un conocimiento de las principales fuentes documentales terciarias en Documentación filosófico-jurídica, véase VILLASEÑOR RODRÍGUEZ, Isabel: "Principales recursos de información para la filosofía jurídica: las guías de fuentes", en RDUNED. Revista Derecho UNED. 2008, $\mathrm{n}^{\circ}$ 3, pp. 261-288.

${ }^{6}$ Véase VILLASEÑOR RODRÍGUEZ, Isabel: "La cátedra de bibliografía jurídica de la Universidad Central de Madrid (1883-1936)", en Revista general de información y documentación, vol. 16, $\mathrm{n}^{\circ} 2$, 2006, pp. 65-91.

${ }^{7}$ BOTREL, Jean François: La diffusion du livre en Espagne (1868-1914): Les libraires. Madrid: Casa de Velázquez, 1988, p.181.

${ }^{8}$ Véase CORDÓN GARCÍA, José Antonio: El registro de la memoria: las bibliografías nacionales y el depósito legal. Gijón: Trea, 1997. 
mitad del siglo XIX ${ }^{9}$ con la revolución industrial (nuevas técnicas de impresión y comercialización) se hace difícil de controlar, así como la producción en nuevos soportes (finales de los años 80 en España).

En cuanto al Derecho, y según Alvite, su período de gran desarrollo y, consecuentemente, el de la Documentación jurídica en general, podemos situarlo en el paso del Antiguo Régimen al Estado moderno ya que se crean instituciones que producen este tipo de Documentación y se estructura la Administración pública, coincidiendo este momento histórico con la denominada "gran era de la codificación, en la que se atiende a una doble finalidad: la recopilación y la estructuración del Derecho vigente. La publicación jurídica se transformó drásticamente $\mathrm{y}$, sin notorias diferencias, ha llegado hasta principios del siglo XXI"10. De esta manera, aflora el mundo editorial jurídico dedicado, fundamentalmente, a dos tipos de publicaciones: los repertorios, es decir, las colecciones exhaustivas de legislación o jurisprudencia, y las revistas jurídicas, que recogerán las aportaciones de autores sobre el Derecho, esto es, la denominada doctrina jurídica. Así, según indica Alvite, en nuestro país se producen anualmente innumerables documentos jurídicos, entre los que se encuentran los que resultan de interés para la Documentación filosófico-jurídica. La misma Alvite dice de ella que "constituye una parte importante de la producción editorial española", tratándose "de un sector dinámico, con una larga tradición y que ha aprovechado, desde los primeros momentos, las ventajas de la web para la difusión y venta de sus productos" $"$. En este sentido también se pronuncia Medina cuando dice que la explosión documental experimentada en el mundo del Derecho "es constatable tanto a nivel de documentos legales, provocada según expertos por la cada vez mayor intervención del Estado en la vida social y económica, como a nivel de bibliografía doctrinal"12.

Por otro lado, una gran parte de la producción bibliográfica que resulta de enorme interés para los usuarios de la Documentación filosófico-jurídica, la "literatura gris", no ha contado con la atención necesaria para poder ser controlada.

En el inicio del siglo XX, los cambios técnicos y el aumento de la alfabetización estimulan el nacimiento de nuevos públicos y diversificación de lectores $\mathrm{y}$, consecuentemente, la multiplicación de documentos (producción masiva) tales como libros escolares, publicaciones técnicas, libros políticos y de pensamiento. Pero aún los lectores son minoría, y en esa minoría se encuentran los usuarios de la Documentación que analizamos.

${ }^{9}$ Botrel, en la obra citada, habla de 500 títulos en 1870 , 1000 en 1880, más de 2000 desde 1905; periódicos: 521 en 1868, cerca de 2000 en 1913.

${ }^{10}$ ALVITE DÍEZ, María Luisa: "Evolución de las bases de datos jurídicas en España", en Anales de documentación, 7, 2004, p.7.

${ }^{11}$ Op. cit., p. 8.

${ }^{12}$ MEDINA ENCINA, Matilde: "Criterios de organización de un centro de documentación jurídica", en Documentación de las ciencias de la información, $\mathrm{n}^{\circ}$ 17, 1994, pp. 241-247. 


\section{LAS FUENTES DE INFORMACIÓN DOCUMENTALES PRIMARIAS}

Podemos decir que la Documentación que es objeto de nuestro estudio se desarrolló fundamentalmente en la segunda mitad del siglo XIX y utilizando como vehículo la monografía, y más concretamente el manual o libro de texto con fines de ayuda a la docencia universitaria. Analizar los libros de texto nos permite conocer el nivel de desarrollo de las distintas disciplinas científicas y, en este sentido, se puede afirmar que España vivía cierto retraso con respecto a las grandes potencias científicas europeas de principios del siglo XIX: Francia, Reino Unido y Alemania.

En relación con este tema conviene tener en cuenta que la implantación y desarrollo de las asignaturas filosófico-jurídicas en la Universidad española como disciplinas académicas no estuvieron exentos de problemas. Desde que en 1770, y como producto de las ideas ilustradas, Carlos III instaurara la primera cátedra de Derecho Natural y de Gentes en los Reales Estudios de San Isidro, las disciplinas relacionadas con la Documentación objeto de nuestro interés han sufrido continuos cambios de nombre ${ }^{13} \mathrm{y}$ de contenido, suprimiéndose incluso durante algún tiempo ${ }^{14}$. Puede decirse que la implantación $^{15}$, en 1850, en el Doctorado de la Universidad Central (Madrid) de la asignatura Filosofia del Derecho ${ }^{16}$ junto con la reforma de Gamazo en 1883, que sustituye la asignatura de Prolegómenos del Derecho ${ }^{17}$ por la de Principios de Derecho Natural, determinó el asentamiento de las dos asignaturas más estrechamente relacionadas con la Documentación que analizamos ${ }^{18}$.

$\mathrm{Y}$ así, la producción de manuales relacionados con dichas asignaturas empieza a desarrollarse en la segunda mitad del siglo XIX; manuales que harán las veces de obras

${ }^{13}$ Derecho Natural y de Gentes (1770), Principios de Legislación Universal (1821), Prolegómenos de Derecho (1842), Legislación Comparada (1845), Filosofia del Derecho (1850), Introducción al estudio del Derecho y Principios de Derecho Natural (1858), Principios de Derecho Natural (1883) y Elementos de Derecho Natural (1884).

${ }^{14}$ Una Orden de Carlos IV de 1794 prohíbe la enseñanza de estas disciplinas en todas las Universidades, seminarios y colegios del Reino.

${ }^{15}$ Según JOSÉ M ${ }^{\mathrm{a}}$. PÉREZ-PRENDES MUÑOZ-ARRACO (Crónica histórica de la Facultad de Derecho. Madrid: Universidad Complutense de Madrid, Facultad de Derecho, 2000) y ANTONIO JIMÉNEZ-LANDI MARTÍNEZ (Breve historia de la Institución Libre de Enseñanza. Sevilla: Junta de Andalucía, Consejería de Educación y Ciencia; Fundación Monte, 1998) fue Julián Sanz del Río (18141869. Profesor de Filosofía), introductor de la filosofía de Krause en España, quien propuso, en 1842, la creación de una cátedra de Filosofía del Derecho en la Universidad Central.

${ }^{16}$ La asignatura Filosofia del Derecho y Derecho Internacional se impartía en Doctorados de otras universidades. En la de Oviedo y Santiago hasta 1875; en la de Barcelona, durante los cursos de 1869-70 y 1870-71; en la Universidad Libre de Vitoria entre 1869 y 1873 y gracias a las leyes de libertad de enseñanza promulgadas por la "Septembrina", se desarrollaban estudios de Doctorado. Un Decreto Gubernamental de 28 del 10 de 1869 suprime las Universidades libres.

17 Aunque podría pensarse que no llega a ser una asignatura iusfilosófica, supone un claro antecedente de las asignaturas Filosofia del Derecho y Derecho Natural, a pesar de que consistiese en una explicación introductoria de los conceptos fundamentales que los estudiantes verían a lo largo de la carrera.

${ }^{18}$ A destacar el año 1887, fecha en la que todas las cátedras de Derecho Natural de las Universidades tienen titular o están pendientes de la celebración de la oposición. 
de consulta. Riaza considera a los manuales como "literatura jurídica popular" y dice que están "destinados a aquellas personas que no estando especializadas en el manejo directo de las fuentes jurídicas necesitaban en ocasiones de precisar el sentido de algún término o conocer el contenido de una institución" ${ }^{19}$. Por su parte, Martínez Neira ${ }^{20}$ afirma que los libros de texto y manuales universitarios en Derecho (y otras disciplinas) nacen con la Ilustración y su racionalismo. Antes, los estudios jurídicos se hacían directamente sobre los textos romanos o canónicos. Se trataba de eliminar el sistema de apuntes, que era considerado como decadente, y de obligar a los profesores a no dictar sino a desarrollar su explicación de viva voz y basándose en unos autores recomendados en los planes de estudio cuyos libros debían comprar los alumnos y aprender de memoria. Aunque había algún precedente, Carlos III empezó a usarlos en su programa de reformas y en los planes de estudio se empezaron a incluir los autores por los que debían estudiarse cada una de las materias que se impartían en una facultad. Aunque la intención era ofrecer textos únicos, con frecuencia aparecían varios, sobre todo por la ausencia de un libro adecuado. Según este autor, podemos decir "que fueron los ilustrados los primeros que comprendieron toda la potencialidad que tenía la utilización de los manuales en la universidad" ya que "el manual permitía al poder uniformar la ciencia, es decir, establecer unos mínimos, un método, un sistema, una doctrina fácil y eficazmente". Los libros de texto son "esenciales para los liberales a la hora de plantearse los problemas de la universidad y su solución" ${ }^{21}$ para dar unidad a la enseñanza, elevar el nivel de la superior y como medio clave para las reformas de la institución universitaria. Por eso, desde 1845 a 1868 se dio en nuestro país una experiencia interesante como es la de las listas de textos como sistema alternativo al del texto único o al de la libertad de texto ${ }^{22}$. Se propone una lista de obras entre las que puede elegir el catedrático y se da el caso de que, ante la escasa variedad de este tipo de obras y españolas ${ }^{23}$, se anime a la redacción y edición de las mismas concediendo

19 RIAZA MARTÍNEZ-OSORIO, Román: Historia de la literatura jurídica española. Madrid: Universidad Complutense, 1998, p. 97. Podemos decir que Román Riaza es el autor del único estudio monográfico sobre un repertorio: "Sobre La Peregrina y sus redacciones" ( Anuario de Historia del Derecho Español, VII, 1930, pp.168-182).

${ }^{20}$ MARTÍNEZ NEIRA, Manuel: El estudio del derecho. Libros de texto y planes de estudio en la Universidad contemporánea. Madrid: Instituto Antonio de Nebrija de estudios sobre la Universidad, Universidad Carlos III, Dykinson, 2001 (Biblioteca del Instituto Antonio de Nebrija de estudios sobre la Universidad, 4). VÉASE también MARTÍNEZ NEIRA, Manuel: "Lecturas antiguas y lecturas ilustradas. Una aproximación a los primeros manuales jurídicos", en Cuadernos del Instituto Antonio de Nebrija, 1, 1998, p. 143-209 y GARCÍA TROBAT, Pilar: "Libertad de cátedra y manuales en la facultad de derecho (1845-1868)", en Cuadernos del Instituto Antonio de Nebrija, 2, 1999, p.37-58.

${ }^{21}$ Op. cit., p. 17 y 15 respectivamente.

${ }^{22}$ Es el llamado Plan Pidal (Real Decreto de 17 de septiembre de 1845) el que propone por primera vez este sistema. Por su parte, el Decreto 21 de Octubre de 1868 determina la absoluta libertad de textos, aunque hubo un intento posterior de recuperar las listas (Decreto 26 de Febrero de 1875) que no prosperó.

${ }^{23}$ En los años 30 y 40 se utilizaban las traducciones de autores tales como Heineccio (1837), Burlamaqui (1838), Vattel (1840) o Félice (1841). Una traducción significativa fue la del Curso de Derecho Natural o Filosofía del Derecho formado con arreglo al estado de esta ciencia en Alemania de Enrique Ahrens a cargo de Ruperto Navarro Zamorano publicada en 1841 porque Ahrens es un pensador 
premios para ello e incluso recomendaciones sobre su contenido (lo elemental de la materia que es objeto de la asignatura) y su forma (claridad, exactitud, de extensión proporcionada) ${ }^{24}$.

Estas listas no se utilizaban para el Doctorado, donde se permitía la libertad de texto aunque debía darse a conocer al Gobierno cuál se iba a utilizar. Tenían una función pedagógica, ideológica y económica. En la actualidad sirven como fuente de conocimiento de toda una ciencia jurídica y de la bibliografía que formó a varias generaciones de juristas, mostrándonos la bibliografía corriente en nuestro país. Es de lamentar que las descripciones de los documentos incluidos en ellas fueran a veces tan incompletas al responder a una información aproximada que conocían los destinatarios. De las asignaturas que nos interesan tan sólo tenemos noticia de algunos manuales de Derecho natural $^{25}$ que aparecen en las listas que se editaron en ese período de tiempo; no hay noticia de los de Filosofia del Derecho porque esta asignatura se impartía en Doctorado. Esta experiencia duró hasta 1868, fecha en la que se da libertad de texto y cátedra a los profesores. Tan sólo en 1875 existe un intento de restaurarlas, pero fue infructuoso. Rus Rufino afirma que todo profesor de filosofía y de derecho que se preciara publicaba un manual, un estudio monográfico o un folleto sobre el Derecho natural, aunque más en el extranjero (ya en los siglos XVII y XVIII) que en España (siglo XIX). Los autores españoles no llevan a cabo "realizaciones" tan importantes ni tan numerosas, "pero sí demuestran - afirma el autor- que se participó en el debate intelectual generado por el Derecho Natural y se trataron los aspectos fundamentales que constituía la nueva ciencia" ${ }^{26}$. Según este autor, con la publicación en 1884 de un manual $^{27}$ que lleva por título Elementos de Derecho natural, "se abre la serie de tratados de derecho natural con este nombre" 28 . No todos los profesores escribieron sus manuales, aunque algunos docentes ${ }^{29}$ sí lo hicieron, para ayuda de los alumnos.

Torres Campos subraya la necesidad e importancia de los tratados "como condición de la adquisición de conocimiento" ${ }^{30}$. Para este autor no puede existir otro medio para

krausista y a través de él se va a conocer el pensamiento de Krause y esta corriente va a tener mucho peso hasta bien entrado el siglo XX (1936) en la Facultad de Derecho y en muchos de sus profesores que, al margen de sus ideologías, están de acuerdo en la necesidad de renovación en la Universidad.

${ }^{24}$ Plan Pidal.

${ }^{25}$ Los de Gómez de la Serna, Falck, Álvarez Martínez y Miquel, por ejemplo, junto con otros manuales que se editaron a lo largo del siglo XIX. El de Pou y Ordinas es una obra declarada de utilidad para la enseñanza del Derecho.

${ }^{26}$ RUS RUFINO, Salvador: "La enseñanza del derecho natural en el último tercio del siglo XIX", en Manuales y textos de enseñanza en la universidad liberal. VII Congreso internacional sobre la historia de las universidades hispánicas. Madrid: Instituto Antonio de Lebrija de estudios sobre la Universidad, Universidad Carlos III de Madrid, 2004, p.656.

${ }^{27}$ El autor es J. Mendive, y se publica en Valladolid, en la imprenta de la viuda de Cuesta.

${ }^{28}$ Op. cit., p.661.

${ }^{29}$ Es el caso de Alfredo Brañas, Rafael Rodríguez de Cepeda, Francisco Javier González Castejón y Luis Mendizábal Martín, entre otros, profesores destacados que ejercieron su docencia a finales del siglo XIX y principios del XX.

${ }^{30}$ Estudios de Bibliografía española y extranjera del Derecho y del Notariado. Madrid: Academia Matritense del Notariado, Establecimiento Tipográfico de Eduardo Cuesta, 1878, p.3. 
llegar al conocimiento de la Ciencia que el estudio de los tratados en unión con la meditación y las investigaciones personales. En este sentido cabe destacar lo que dice en relación con la Ciencia en España y en el siglo XIX, que transcribimos a continuación:

\begin{abstract}
"España ha quedado, por desgracia, rezagada, merced a su malestar interior, a sus guerras y a sus conmociones continuas, en el movimiento europeo. Hasta cerca de la segunda mitad del siglo, no han penetrado en nuestro país investigaciones generalizadas ya mucho antes. La poca consideración en que se ha tenido el Profesorado, mezquinamente retribuido, y la afición a la Política, que estiriliza las inteligencias mejores, son también causas que explican cumplidamente nuestro atraso" ${ }^{31}$.
\end{abstract}

Por último, y según Gil Cremades ${ }^{32}$, este género de literatura didáctica se prodiga en la inmediata postguerra española aunque durante años escaseen las nuevas ediciones y se reediten los mismos manuales continuamente.

En relación con el currículo académico se encuentra también otro tipo de fuentes que son de gran interés por su alto valor informativo así como por su contribución al debate científico y por sus extensas aportaciones bibliográficas. Se trata de las tesis doctorales. Pero no es hasta 1847 cuando los estudios de Doctorado culminan con un discurso o tesis. Antes, y sólo a partir de 1812, los sucesivos gobiernos van incorporando en sus reformas educativas variaciones en los planes de estudios universitarios que progresivamente van dando más importancia académica al grado de doctor $^{33}$, importancia que no tenía hasta el momento. Un dato curioso es el hecho de que desde 1845 el Doctorado se centraliza en Madrid y hasta 1954 la tesis se defiende exclusivamente en la Universidad Central. Según Aurora de Miguel ${ }^{34}$, durante los años 50 y 60 del siglo XIX los discursos o tesis no cuentan con originalidad científica ni calidad investigadora porque la Universidad española no investiga en esta etapa ni los profesores universitarios publican trabajos originales de investigación. Y eso, a pesar de la "modernidad" de conocimientos que se impartían en el Doctorado. Es a finales del siglo (años 80) y gracias al impulso precisamente de un catedrático de la asignatura Filosofía del Derecho, Giner de los Ríos ${ }^{35}$, cuando la tesis doctoral se empieza a desarrollar y valorar como un trabajo de investigación. De forma que no es hasta el

${ }^{31}$ Nociones de Bibliografía y Literatura jurídicas de España. Madrid: Góngora, 1884, p.281.

32،"Filosofía del derecho en España (1960-1985)”, en Anales de la Cátedra Francisco Suárez, 1985, $\mathrm{n}^{\circ} 25$, pp.225-243.

${ }_{33}$ En el Antiguo Régimen existían 3 Facultades Mayores (Teología, Cánones y Leyes y Medicina) y 3 grados: bachiller, licenciado y doctor, de los cuales el primero habilitaba para la práctica profesional y los otros dos se estructuraban para la carrera académica. No es hasta 1843 que aparecen por primera vez los estudios de Doctorado con cursos específicos, tal y como hoy se conoce.

${ }^{34}$ MIGUEL ALONSO, Aurora:" Los estudios de doctorado y el inicio de la tesis doctoral en España. 1847-1900". Separata de Archivos universitarios e Historia de las Universidades, 9, 2003, pp.197-222

${ }^{35}$ Francisco Giner de los Ríos (1839-1915) se hizo cargo de la cátedra de Doctorado en 1866, renunciando a ella un año después en solidaridad con su maestro Julián Sanz del Río y otros compañeros. Se reincorpora en 1868 hasta 1875 , fecha en la que se le separa de la cátedra por motivos políticos, y vuelve a ella en 1881. 
siglo XX cuando la tesis empieza a ser lo que es ahora: un trabajo original de investigación que culmina el tercer ciclo de la enseñanza universitaria.

Según la misma autora, las fuentes donde podemos encontrar tesis del siglo XIX son el Archivo Histórico de la Universidad Complutense de Madrid (la primera tesis con la que cuenta es de 1887), el Archivo Histórico Nacional y las Bibliotecas de cada Facultad.

De todos es sabido que el siglo XIX español es un siglo convulso, repleto de actividad política y parlamentaria. Y eso se refleja también en el campo de la Documentación. La mayor parte de los pensadores y profesores universitarios forman parte de instituciones donde se debaten e intercambian ideas. De ahí que, según hemos podido comprobar, se pueda decir que, en cuanto a publicación científica se refiere, el siglo XIX español es fundamentalmente un siglo de manuales, discursos y folletos más que de ensayos o, incluso, artículos en revistas científicas. Discursos de apertura de curso en la Universidad o en las múltiples instituciones "culturales" que proliferan a finales de siglo, de conmemoraciones, de ingresos en Academias; un siglo de memorias, de actas, de informes de sesiones o textos que transcriben las discusiones habidas en el seno de alguna sociedad científica. Ejemplos todos ellos de lo que se ha dado en llamar "literatura gris" y vehículos notables de información y comunicación científica. Jean François Botrel ${ }^{36}$ afirma que hacia la segunda mitad del siglo XIX, cuando se produce lo que ha dado en llamar la "segunda revolución de la imprenta", se da una masificación de producción de impresos tales como actas, memorias, estatutos, discursos, etc que, "por carecer de importancia mercantil no se incluyeron en las bibliografías corrientes pero que, año tras año, fueron constituyendo el patrimonio y la memoria de la sociedad española de que tanto periódicos como revistas, más o menos duraderos, dieron fe por todo el territorio". Documentación de gran valor de la que tenemos escasas noticias al escapar de ese control bibliográfico ${ }^{37}$ del que, aún hoy en día, escapan.

Otro vehículo de difusión y comunicación de conocimiento científico a gran escala es el que representan las publicaciones periódicas, y más concretamente las revistas que, como se sabe, se dan desde el siglo XVII ${ }^{38}$. Se trata de fuentes que responden a la necesidad de difundir unos conocimientos periódicamente, con cierta continuidad, de carácter colectivo y propias de una originalidad en la información que transmiten de gran interés para sociedades científicas y estudiosos en general. Es cierto que en la materia que nos ocupa o materias cercanas no se desarrollan totalmente hasta el siglo $\mathrm{XX}$, pero ya en el siglo XIX contamos con algunos ejemplos. Uno de ellos lo tenemos en el Boletín de la Institución Libre de Enseñanza, que contó hasta 1889 y entre sus

\footnotetext{
36 "Para una estadística bibliográfica de la España contemporánea. Reflexiones y sugerencias", en Varia bibliográfica: Homenaje a José Simón Díaz. Kassel: Reichenberger, 1988, p.106.

${ }^{37}$ Son muchos los autores que se han lamentado y lamentan de los problemas planteados por el escaso control bibliográfico que, a pesar de disposiciones legislativas creadas al efecto, se ha dado en nuestro país. Para más detalle véase CORDÓN GARCÍA, José Antonio: El registro de la memoria: las bibliografías nacionales y el depósito legal. Gijón: Trea, 1997.

${ }^{38}$ Sobre este asunto ver LÓPEZ YEPES, José: "La publicación periódica de carácter científico como medio de información documental. Origen y evolución histórica", en Fundamentos de Información y Documentación. Madrid: EUDEMA, 1989, pp. 101-131.
} 
secciones con las de Filosofía y Derecho natural. Entre sus páginas se encuentran trabajos de los profesores de la Institución, críticas de libros e investigaciones, extractos de las lecciones de los cursos superiores y noticias sobre sus actividades. También contamos con publicaciones periódicas emanadas de organismos culturales tales como las reales academias y así tenemos la Revista de la Real Academia de Jurisprudencia y Legislación (1875) o el Anuario de la Real Academia de Ciencias Morales y Políticas (1859) y los Anales de la Real Academia de Ciencias Morales y Políticas (1934).

En la actualidad se ha desarrollado la publicación de revistas en soporte informático, $\mathrm{y}$, aunque no demasiados, podemos contar con algún ejemplo de publicación especializada en nuestro campo que se ofrece en dos soportes: el clásico y el electrónico. Así ocurre con los Cuadernos electrónicos de Filosofía del Derecho (1998)$^{39}$, que es la versión electrónica de la revista Anuario de filosofía del derecho, publicación oficial de la Sociedad Española de Filosofía jurídica y política o El basilisco (http://filosofia.org/rev/bas), también en papel.

En relación con las revistas, otra publicación periódica es el diario o periódico, que tuvo gran importancia en el siglo XIX (segunda mitad) y en nuestro país como medio de difusión de las sociedades científicas y culturales, hasta el punto de que daban cuenta de las sesiones habidas en organizaciones tales como el Ateneo de Madrid. De ahí el enorme interés que tienen para la Documentación objeto de nuestro estudio, ya que resultaban ser vehículo de difusión de estudiosos de las ideas, filósofos que exponían sus teorías y análisis sólo en las aulas o en los salones de esas instituciones. En los años finales del siglo XIX y primeras décadas del siglo XX nacen los diarios más famosos del periodismo español. La burguesía urbana era el principal grupo social lector de periódicos. Será en la segunda década del siglo XX cuando aumenten el número de páginas (de 4 a 6 u 8). Se daban dos variedades: la prensa de empresa (ABC, El Debate, $E l \mathrm{Sol}^{40}$ ) frente a pequeños periódicos de contenido exclusivamente político (la mayoría y, sobre todo en el período de 1854 a 1874). En la segunda mitad del siglo XIX se daba el periódico de opinión, con artículos políticos, ideológicos, doctrinales, siendo habitualmente la voz de un partido político. Algunos ejemplos son: La correspondencia de España, El día (liberal), La época (primero liberal, luego moderado y después alfonsino), El globo (republicano), El Heraldo (moderado), La Iberia (monárquico), El imparcial o gaceta política y literaria (adicto al gobierno de José Bonaparte), El liberal, El derecho. Periódico radical o La nación (progresista), entre otros.

A finales del siglo y, sobre todo a partir de 1900, el periodismo cambia en sus planteamientos buscando informar y entretener, ofreciendo la noticia, el reportaje y la entrevista. De la voz de un partido a órgano de información relativamente neutro al servicio de la empresa y los lectores, del periodismo político al profesional. Se trata del resultado de una evolución lenta aunque existen factores que favorecieron la transformación tales como la extensión de la red ferroviaria y telegráfica, la creación de

\footnotetext{
${ }^{39}$ http://www.uv.es/AFD) (http://www.uv.es/CEFD

${ }^{40}$ Una información detallada sobre algunos de estos diarios puede verse en SÁNCHEZ ILLÁN, Juan Carlos: "La edición de periódicos y la empresa periodística", en Historia de la edición en España (18361936). Dirigido por Jesús A. Martínez Martín. Madrid: Marcial Pons, 2001, pp.397-414.
} 
un capitalismo de empresa, los portentosos adelantos técnicos en las máquinas de imprimir que permitían grandes tiradas.

En la actualidad, la generalización de acceso a Internet ha permitido el desarrollo y acceso de la prensa on line. En España, tras un inicio lento, "la progresión de la prensa digital (o electrónica) fue constante y conoció un notable despegue a partir del año $2000^{\prime, 41}$, cuando la mayoría de los periódicos impresos ya estaban en red. Se trata de un nuevo producto, de un nuevo medio de comunicación a veces muy distinto de la prensa escrita, que "facilita más allá de una información instantánea, siempre disponible, una multitud de servicios y conexiones" ${ }^{\prime \prime 2}$. Antes de editar sus primeras páginas web, numerosos diarios españoles iniciaron su andadura electrónica con la comercialización de CD-ROM. Al principio ofrecían en línea los mismos contenidos que la edición impresa; luego empezaron a ofrecer otro formato con mucha imagen, poco texto, actualizaciones de última hora, foros, chats, enlaces, portales de ocio, etc

\section{LAS FUENTES DE INFORMACIÓN DOCUMENTALES SECUNDARIAS}

En cuanto a la experiencia con otro tipo de fuentes documentales en el siglo XIX, podemos decir que no fue nada buena. Entre las fuentes documentales secundarias que contienen información primaria reelaborada, sintetizada y reorganizada, no se puede afirmar que contemos con fuentes de carácter biográfico colectivas, por ejemplo. Llano y Rus dicen al respecto: "A diferencia de otros países, nos encontramos con que en España no hay un diccionario de los juristas del siglo XIX, esto es, un texto en el que se recojan todos los autores que de una u otra forma dedicaron su vida al Derecho en sus diversas ramas" ${ }^{43}$. Son pocos los ejemplos que hemos encontrado en este sentido y la mayoría ofrecen información, bien sobre juristas de todas las ramas, bien sobre pensadores en general. Quizá el repertorio más específico sea el que nos ofrecen los autores mencionados. En él se nos da información bio-bibliográfica de todos los profesores universitarios dedicados a la enseñanza de disciplinas relacionadas con la materia que es objeto de nuestro interés pero se limita al siglo XIX, no existiendo ninguna otra obra que continúe este trabajo.

Por su parte, Gil Cremades ${ }^{44}$ afirma que el primer esbozo de historia de la Filosofía del Derecho en España lo traza Recaséns Siches en 1929, con la publicación de la traducción de un clásico ${ }^{45}$ y haciendo uso de una práctica habitual en nuestro país: la del

\footnotetext{
${ }^{41}$ MOREAU, Marie Christine: "La prensa digital española: la configuración de un nuevo medio", en Prensa, impresos, lectura en el mundo hispánico contemporáneo. Homenaje a Jean-François Botrel. Edición a cargo de Jean-Michel Desvois. Pessac Cedex: PILAR (Prensa, Impresos, Lectura en el Área Románica), 2005, pp.572.

${ }^{42}$ Ibidem.

${ }^{43}$ LLANO TORRES, Ana y RUS RUFINO, Salvador: Historia del pensamiento filosófico y jurídico. La enseñanza de las disciplinas iusfilosóficas en la Universidad española del siglo XIX y sus protagonistas. León: Universidad, 1997, p.12.

${ }_{44}$ Op. cit., p. 230.

${ }^{45}$ VECCHIO, L. del: Filosofia del Derecho. Barcelona: Bosch, 1929.
} 
apéndice que el traductor de una obra básica de autor extranjero incluye en la edición de la traducción a modo de crónica, dando cuenta de autores españoles. El mismo autor señala que también se pueden encontrar referencias sobre autores españoles en publicaciones referidas a la Filosofía o al pensamiento filosófico en general, aunque se lamenta de que éstas sean escasas.

Hemos encontrado algunas obras que recogen información biográfica de los miembros de alguna institución cultural como el Ateneo o las Reales academias de Jurisprudencia y Legislación ${ }^{46}$ o de Ciencias Morales y Políticas ${ }^{47}$, y repertorios genéricos referidos a juristas o pensadores ilustres entre los cuales puede encontrarse alguna información de interés.

En cuanto a diccionarios y otras obras de semejantes características, Barrero, en su estudio sobre los repertorios y diccionarios jurídicos desde la Edad Media hasta nuestros días, califica estos documentos como "obras que se caracterizan por tener una finalidad eminentemente práctica o auxiliar para el manejo de los textos jurídicos y adoptan un criterio alfabético en la ordenación de su contenido" ${ }^{48}$. Se trata de literatura jurídica auxiliar ${ }^{49}$ que en su mayor parte no se imprimió al no estar destinada a la imprenta "por su propia naturaleza". Son obras poco conocidas por los investigadores pese a la importancia que tuvieron en su época y de gran utilidad para el estudio de cualquier institución. Reciben distintos nombres: vocabulario, tabla, repertorio, prontuario, diccionario, y pueden clasificarse, según Barrero, en vocabularios, repertorios y diccionarios jurídicos. Los primeros son obras que aclaran el sentido de las palabras. A partir del siglo XV son menos frecuentes en España porque el significado de los términos jurídicos se aclara en los otros dos tipos, que los sustituyen. Los repertorios "son obras que contienen simples referencias a los textos legales o a la literatura jurídica" ${ }^{50}$ y los diccionarios "son obras que aúnan la técnica del vocabulario (definición) y del repertorio (utilización de los textos legales) pero no limitándose a la mera referencia" ${ }^{51}$.

Durante el siglo XVIII, la Ilustración permitió innovaciones de repertorios o diccionarios jurídicos, concibiéndose éstos como libros que compendian todo el saber de una ciencia. Es un nuevo tipo de diccionario de contenido doctrinal que, desarrollado y perfeccionado, llega hasta nuestros días. En el XIX, desde mediados de siglo, se da importancia a los diccionarios jurídicos concebidos como recopilaciones alfabéticas de

\footnotetext{
${ }^{46}$ Un ejemplo puede ser BREVE noticia de los cuarenta jurisconsultos españoles inscritos en las tres lápidas de la Academia Matritense de Jurisprudencia y Legislación. Madrid: Imp. de Eusebio Aguado, 1857.

${ }^{47}$ Como ejemplo sirva el de NECROLOGÍAS de los señores académicos de número fallecidos desde el $1^{\circ}$ de julio de 1885 escritas y publicadas en virtud de lo que dispone el art.52 del Reglamento de dicha corporación. Madrid: Real Academia de Ciencias Morales y Políticas, 1898.

${ }^{48}$ BARRERO GARCÍA, Ana María: "Los repertorios y diccionarios jurídicos desde la Edad Media hasta nuestros días (Notas para su estudio)", en Anuario de Historia del Derecho español, tomo XLIII, 1973 , p. 312.

${ }^{49}$ La literatura jurídica auxiliar, por su finalidad práctica, ha existido siempre, evolucionando de acuerdo con las necesidades que el conocimiento del Derecho plantea en cada momento.

${ }^{50}$ p.314.

${ }^{51}$ Ibidem.
} 
textos legales ${ }^{52}$ por el valor de la ley como fuente esencial del Derecho moderno y su profusión. Aunque en su elaboración no se introducen nuevas técnicas, las novedades se dan en cuanto al contenido, apareciendo diccionarios especiales para cada rama del Derecho e incorporando jurisprudencia. Los diccionarios especiales limitan su objetivo a recoger las disposiciones sobre cada una de las ramas del Derecho (jurisprudencia, legislación y, algunos, doctrina) y son muy numerosos ${ }^{53}$. Una novedad desde mediados de siglo es la aparición de una serie de diccionarios que, sin olvidar su finalidad primordial de hacer accesible el conocimiento de la legislación vigente y de la jurisprudencia, y centrándose en lo administrativo, acompañan la parte legislativa de una exposición doctrinal y se publican y actualizan hasta fecha reciente. Es el caso de la Enciclopedia española de Derecho y Administración, por ejemplo. Al mismo tiempo se siguen publicando diccionarios doctrinales concebidos como compendios doctrinales que desean hacer llegar a todas las clases sociales el lenguaje del Derecho. En la actualidad, los diccionarios que recogen el Derecho actual no difieren formalmente de los anteriores; se complementan con todo tipo de índices cronológicos y sistemáticos porque tratan de ser completos y suficientes. Los hay que recogen legislación y jurisprudencia sólo; los que añaden una parte doctrinal o los doctrinales exclusivamente. También los destinados a las distintas ramas del Derecho, aunque se dan con menos frecuencia.

Román Riaza asegura que "los definicionarios jurídicos han existido (...) desde la época visigoda", aunque "la idea de recoger al lado de nociones científicas sobre las instituciones jurídicas las fuentes respectivas, in extenso o una mención de ellas, es algo que caracteriza a las primeras enciclopedias jurídicas que se redactan en nuestra Patria" ${ }^{\text {54 }}$ publicadas en la segunda mitad del siglo XVIII y durante el XIX.

De la importancia de las enciclopedias para los usuarios especializados habla Torres Campos cuando dice que "repertorios universales de los conocimientos humanos", "proporcionan con facilidad las noticias que en cada asunto se desean", de forma que, teniendo en cuenta "los progresos de la cultura y las aspiraciones de nuestro tiempo...es menester un libro que suministre a las personas instruidas conocimientos de las diversas ciencias de que pudieran necesitar" ${ }^{\text {"55 }}$. También las hay jurídicas, pero todas ellas presentan un problema y es el de la ordenación alfabética, "en pugna y contradicción con las exigencias científicas". Según el autor, han proliferado en los cincuenta años anteriores a su declaración y da cuenta de lagunas de distintos países. La mayor parte de

\footnotetext{
${ }^{52}$ Aunque desde principios del XIX las leyes se dan a conocer a través de publicaciones estatales (Gaceta de Madrid, Boletín Oficial del Estado) y se reúnen en colecciones oficiales, la proliferación de leyes y las constantes variaciones en las mismas impuestas por los vaivenes políticos, hacen necesaria la realización de diccionarios que faciliten su manejo y recojan las normas vigentes en cada momento.

${ }^{53}$ Torres Campos, en su Bibliografía española contemporánea del Derecho y de la Politica. 18001880: con tres apéndices relativos a la bibliografía extranjera sobre el derecho español, a la hispanoamericana y a la portuguesa-brasileña (Madrid, etc: Librería de Fernando Fe, etc, 1883-1897), da cuenta de más de un centenar.

${ }_{54}$ Op. cit., p. 203.

${ }^{55}$ Op. cit., p. 37.
} 
las enciclopedias publicadas recogen información genérica referida a todas las ramas del Derecho.

En cuanto a fuentes de información documentales secundarias que remiten a otras fuentes, esto es, las fuentes de información bibliográfica, se puede decir que contamos con algunos ejemplos de bibliografías y catálogos de biblioteca del siglo XIX. Las únicas bibliografías de las que disponemos en ese siglo son las de Torres Campos $^{56}$. Él mismo justifica el interés y necesidad de este tipo de fuentes ${ }^{57}$ diciendo que la Bibliografia es "indispensable al hombre de ciencia para llevar un norte seguro en sus trabajos, y que hoy, merced al desarrollo creciente de la misma, y a las múltiples y continuas publicaciones, es más necesaria que nunca" otro medio para llegar al conocimiento de la Ciencia que el estudio de los tratados en unión con la meditación y las investigaciones personales", y que gozan de considerable interés "las publicaciones que se destinen...a señalar las diversas fuentes de consulta en cada asunto de que se trate" ${ }^{\text {"59 }}$. Habla de las bibliografías generales pero considera de mayor interés para "el abogado y el jurisconsulto" "las bibliografias de Derecho", de gran interés para el estudioso de esos temas y "más completas y detalladas, y más idóneas, por consiguiente, que las de carácter general" ${ }^{160}$. Tras hacer historia de ellas (se remonta al siglo XVI) da cuenta de las más significativas publicadas en todo lugar y tiempo.

Según esto, Sánchez de Castro, autor del primer manual de una asignatura de Documentación especializada que se dio en la Facultad de Derecho de la Universidad Central, inicia su libro ${ }^{61}$ afirmando que no existe hasta ese momento una bibliografía jurídica general, a pesar de las publicadas por Torres Campos, que sólo comprenden autores y publicaciones coetáneas. Este mismo autor se refiere a algunos trabajos de carácter bibliográfico que se encuentran inéditos, "encerrados en los estantes de manuscritos" de la Biblioteca Nacional, como es el caso de la Biblioteca jurídica de España de Fernández Llamazares ${ }^{62}$. Delgado Casado $^{63}$ describe esta bibliografía

${ }^{56}$ Estudios de Bibliografía española y extranjera del Derecho y del Notariado. Madrid: Academia Matritense del Notariado, Establecimiento Tipográfico de Eduardo Cuesta, 1878 (memoria premiada en 1876 en el certamen público de la Academia Matritense del Notariado); Bibliografía española contemporánea del Derecho y de la Política. 1800-1880: con tres apéndices relativos a la bibliografía extranjera sobre el derecho español, a la hispanoamericana y a la portuguesa-brasileña. Madrid (etc): Librería de Fernando Fe (etc), 1883-1897.

${ }^{57}$ En Estudios..., no sólo habla del gran número de tratados de Bibliografía publicados en el siglo XIX sino también de distintas bibliografías, de las que da su opinión, y de la evolución histórica de esta ciencia.

${ }^{58}$ Ibidem, p. 4.

59 Ibidem, p. 6.

${ }^{60}$ Ibidem, p. 17.

61 SÁNCHEZ DE CASTRO, FRANCISCO: Apuntes de literatura y bibliografia jurídicas de España. Madrid: Imprenta de los señores Lezcano y Co., 1883.

62 GÓMEZ DE SALAZAR Y ALONSO da cuenta de ello ("Los premios bibliográficos de la Biblioteca Nacional" en Boletín de la Dirección General de Archivos y Bibliotecas, IV, 1955, 27, p. Azules).

${ }^{63}$ DELGADO CASADO, Juan: Un siglo de Bibliografía en España: los concursos bibliográficos de la Biblioteca Nacional (1857-1953). Madrid: Ollero-Ramos, 2001, p. 396. 
indicando que se inicia con un breve preámbulo sobre la importancia de la bibliografía jurídica de incómoda lectura por el tipo de letra y tinta utilizadas. Se trata de una bibliografía que se presentó al concurso bibliográfico de la Biblioteca Nacional en 1861 y que fue propuesta para su adquisición. Se encuentra en esa institución con la signatura Mss.5609.

Según Sánchez de Castro, lo único que existe entonces son trabajos particulares tales como biografías y monografías. Riaza asegura que "la bibliografía general es escasa e incompleta" "64 y da como referencia la bibliografía de Nicolás Antonio, y los manuales de Ureña y Torres Campos, llamándolos "repertorios bibliográficos". Al igual que ellos, Ureña también se preocupa por el tema. Curiosamente son los profesores de una asignatura destinada a formar a los alumnos de la Facultad de Derecho en el manejo de las fuentes. Según su testimonio apenas existen bibliografías especializadas en Derecho y mucho menos en la materia que nos ocupa.

Botre $^{65}$, al hablar de los libreros especializados en libro antiguo, afirma que hasta principios del siglo XX (años 1915, 1918...) no cuentan con obras de consulta, tan sólo con la bibliografía de Nicolás Antonio, la de Gallardo y el catálogo de la biblioteca de Salvá, todas ellas de carácter general como las bibliografías a las que se refiere cuando asegura que a partir de 1885 y hasta 1905 se produce la eclosión, fomentada por los premios concedidos por la Biblioteca Nacional ${ }^{66}$, de bibliografías preocupadas por dar cuenta de la producción editorial española desde los comienzos de la imprenta aunque centrándose en una ciudad o región fundamentalmente: las tipobibliografías.

El siglo XX, en su evolución, irá procurando bibliografías cada vez más especializadas. Y así, en el campo que nos ocupa nos encontramos con publicaciones dedicadas a recoger la bibliografía sobre asuntos muy específicos. Una de las primeras puede decirse que es la de Modesto Saavedra, que lleva por título "Bibliografía de la Filosofía del Derecho en España (1961-1971)" y fue publicada en 1972 como artículo de la revista Anales de la cátedra Francisco Suárez ${ }^{67}$.

En lo que se refiere a los catálogos de biblioteca publicados, tenemos algunos ejemplos ya en el siglo XIX que pueden ser de interés para los usuarios de la Documentación iusfilosófica por recoger información de los fondos de algunas instituciones donde desarrollaron buena parte de sus actividades académicas y profesionales pensadores del Derecho. Los catálogos de las Reales Academias de Jurisprudencia y Legislación ${ }^{68}$ o de Ciencias Morales y Políticas así como el del Ateneo de Madrid o los de los Colegios de Abogados de Madrid y Barcelona, los del Congreso,

${ }^{64}$ Historia de la literatura jurídica española. Notas de un curso, por-----Profesor Auxiliar de la asignatura en la Universidad Central, y Catedrático, excedente, de Historia del Derecho Español. Madrid: Litografía E. Nieto, 1930, p.28.

${ }_{65}^{65}$ La diffusion du livre en Espagne (1868-1914): Les libraires. Madrid: Casa de Velázquez, 1988.

${ }^{66}$ Para conocer más completa información sobre este asunto, véase la obra citada de Juan DELGADO CASADO.

${ }^{67} \mathrm{n}^{\mathrm{o}} 12, \mathrm{v} .2$, pp. 161-200.

${ }^{68}$ El que hizo Torres Campos, por ejemplo, cuando fue su bibliotecario: Catálogo sistemático de las obras existentes en la Biblioteca de la Academia de Jurisprudencia y Legislación. Madrid: Impr. del Ministerio de Gracia y Justicia, 1876. 
el Senado, el Ministerio de Gracia y Justicia o del Consejo de Estado, y tantos otros que hoy se mantienen actualizados en soporte informático.

Aurora Miguel $^{69}$ informa de que los catálogos de la Facultad de Teología y Jurisprudencia (Universidad de Alcalá de Henares) se empezaron a redactar en 1848 pero que en 1915, en un informe referido a la biblioteca de la Facultad de Derecho, se alude a ellos en términos de pasado. Se trata de catálogos por materias, en hojas sueltas y organizados según la clasificación de Brunet. De ellos se conservan 39 tomos en los que ya no se incluyen libros con años de impresión posterior a la década de los sesenta. De todos conviene destacar un volumen de la sección de Jurisprudencia (de Derecho natural y de gentes) así como los 2 volúmenes de Ciencias filosóficas y morales de Otras secciones.

En la actualidad, debemos destacar, además de los mencionados, los catálogos de las bibliotecas universitarias, que, on line, nos ofrecen información general en sus páginas, no sólo de los fondos contenidos en ellos, sino también de los específicos de cada facultad, pudiendo acceder a su información desde cualquier parte del mundo.

Por otra parte, el mismo Botrel ${ }^{70}$ nos informa de la actividad de los libreros en la publicación de catálogos comerciales tanto de fondo antiguo como moderno, y que se inicia hacia finales del siglo XIX (a partir de los años 60-70). Son obra de profesionales que, con mayor o menor exhaustividad en sus descripciones, dan cuenta de los productos que tienen a la venta. Esta es una actividad que se ha ampliado y perfeccionado con el tiempo, aunque en el primer tercio del siglo XX se editaron catálogos muy detallados, con ilustraciones, fotografías, sugerencias de lectura...respondiendo a la importancia que se les daba como seña de identidad de las casas editoriales y vehículo publicitario. En la actualidad, debemos destacar el desarrollo experimentado por editoriales y librerías jurídicas gracias a la aplicación de las nuevas tecnologías. Internet les ha permitido desarrollar productos que, no sólo informan de sus novedades y permiten la compra en el momento, sino que también ofrecen todo tipo de información de interés para sus clientes a partir de enlaces ${ }^{71}$.

\section{CONCLUSIONES}

Tras el análisis realizado del desarrollo experimentado por algunas de las fuentes de información documentales utilizadas y creadas por usuarios de la Documentación filosófico-jurídica, podemos decir que, en los siglos estudiados, la producción de las mismas es amplia aunque existe poco control sobre ella. Empezó a desarrollarse fundamentalmente durante la segunda mitad del siglo XIX utilizando como vehículo la

\footnotetext{
69 "Del Plan Pidal a la Ley Moyano: consolidación de la Biblioteca de la Universidad Central”, en Estudios históricos. Homenaje a los profesores José Ma Jover Zamora y Vicente Palacio Atard. Madrid: UCM, Departamento de Historia Contemporánea, 1990, pp. 681-701.

${ }^{70}$ Op.cit.

${ }^{71}$ Sirvan como ejemplos las siguientes referencias: Editorial Cívitas (http://www.civitas.es), Librería Dykinson (http://www.dykinson.com), Librería Marcial Pons (http://www.marcialpons.es).
} 
monografía, y más concretamente el manual o libro de texto con fines de ayuda a la docencia universitaria. Junto a este tipo, la "literatura gris" tuvo gran desarrollo en esa misma época, sobre todo aquellos documentos emanados de instituciones académicas o culturales $^{72}$, donde la mayor parte de pensadores y profesores universitarios debaten e intercambian ideas. Esta tradición se ve interrumpida en siglos posteriores, donde se desarrollan otras formas de Documentación, como puede ser la revista y el ensayo o la tesis doctoral, y de comunicación científica, pasando de la relación entre colegas desarrollada con intensidad en el siglo XIX a la institucionalización, en la segunda mitad del siglo XX, en congresos, y la comunicación a través de otros medios técnicos.

También se puede afirmar que las fuentes documentales secundarias en Documentación iusfilosófica no han experimentado un gran desarrollo. De las que ofrecen información biográfica, la primera fuente específica no la encontraremos hasta la obra de Llano Torres y Rus Rufino, en 1997. Lo mismo ocurre con las bibliografías, de las que tenemos en el siglo XIX dos ejemplos de bibliografías dedicadas al Derecho en general en las obras de Torres Campos, y ya en 1972 una especializada en Filosofía jurídica. En cuanto a catálogos de biblioteca debemos destacar la interesante actividad que se desarrolló durante la segunda mitad del siglo XIX en la edición de los de algunas bibliotecas especializadas en Derecho y de interés para los usuarios de la Documentación iusfilosófica. Por su parte, en relación con diccionarios y enciclopedias, podemos decir que los primeros, aunque se dan ya desde la Edad Media, es en la segunda mitad del siglo XIX cuando se desarrollan los específicos para todas las ramas del Derecho, y a comienzos del siglo XXI los especializados en Filosofía jurídica ${ }^{73}$. No ocurre así con las enciclopedias, que siguen publicándose y ofreciendo una información de carácter genérico e introductorio sobre todas las ramas del Derecho.

Respecto a las fuentes de información documentales terciarias específicas de Documentación iusfilosófica no se crean hasta finales del siglo XX con la aparición de portales en Internet tales como Doxa o filosofiayderecho.com.

\section{REFERENCIAS BIBLIOGRÁFICAS}

ALVITE DÍEZ, María Luisa: "Evolución de las bases de datos jurídicas en España”, en Anales de documentación, 7, 2004, pp.7-27

BARRERO GARCÍA, Ana María: "Los repertorios y diccionarios jurídicos desde la Edad Media hasta nuestros días (Notas para su estudio)", en Anuario de Historia del Derecho español, tomo XLIII, 1973, pp.311-351

${ }^{72}$ Un estudio de las instituciones de mayor significación para los usuarios de la Documentación iusfilosófica se encuentra en VILLASEÑOR RODRÍGUEZ, Isabel: "Instituciones de interés para la Filosofía jurídica como fuentes de información", en Revista General de información y documentación. Universidad Complutense de Madrid, 2009, v. 19, pp. 297-316.

${ }^{73}$ DICCIONARIO jurídico: filosofía y teoría del derecho e informática jurídica. Antonio Enrique Pérez-Luño, Ramón Luís Soriano Díaz, Carmelo José Gómez Torres (directores de la edición). Granada: Comares, 2004 
BOTREL, Jean François: La diffusion du livre en Espagne (1868-1914): Les libraires. Madrid: Casa de Velázquez, 1988

BOTREL, Jean François: "Para una estadística bibliográfica de la España contemporánea. Reflexiones y sugerencias", en Varia bibliográfica: Homenaje a José Simón Díaz. Kassel: Reichenberger, 1988

BOTREL, Jean François: Libros, prensa y lectura en la España del siglo XIX. Madrid (etc): Fundación Germán Sánchez Ruipérez; Madrid: Pirámide, D.L.1993

CORDÓN GARCÍA, José Antonio: El registro de la memoria: las bibliografias nacionales y el depósito legal. Gijón: Trea, 1997

DELGADO CASADO, Juan: Un siglo de Bibliografia en España: los concursos bibliográficos de la Biblioteca Nacional (1857-1953). Madrid: Ollero-Ramos, 2001

GARCÍA TROBAT, Pilar: "Libertad de cátedra y manuales en la facultad de derecho (1845-1868)", en Cuadernos del Instituto Antonio de Nebrija, 2, 1999, pp.37-58

GIL CREMADES, Juan José: "Filosofía del derecho en España (1960-1985)", en Anales de la Cátedra Francisco Suárez, 1985, n²5, pp.225-243

GÓMEZ DE SALAZAR Y ALONSO, Julio: "Los premios bibliográficos de la Biblioteca Nacional”, en Boletín de la Dirección General de Archivos y Bibliotecas, IV, 1955, 27, páginas azules

JIMÉNEZ-LANDI MARTÍNEZ, Antonio: Breve historia de la Institución Libre de Enseñanza. Sevilla: Junta de Andalucía, Consejería de Educación y Ciencia; Fundación Monte, 1998

LLANO TORRES, Ana y RUS RUFINO, Salvador: Historia del pensamiento filosófico y jurídico. La enseñanza de las disciplinas iusfilosóficas en la Universidad española del siglo XIX y sus protagonistas. León: Universidad, 1997

LÓPEZ YEPES, José: "La publicación periódica de carácter científico como medio de información documental. Origen y evolución histórica", en Fundamentos de Información y Documentación. Madrid: EUDEMA, 1989, pp. 101-131

MANUAL de documentación jurídica. Edit. Por Mateo Maciá. Madrid: Síntesis, 1998

MANUALES y textos de enseñanza en la universidad liberal. VII Congreso internacional sobre la historia de las universidades hispánicas. Madrid: Instituto Antonio de Lebrija de estudios sobre la Universidad, universidad Carlos III de Madrid, 2004

MARTÍNEZ NEIRA, Manuel: "Lecturas antiguas y lecturas ilustradas. Una aproximación a los primeros manuales jurídicos", en Cuadernos del Instituto Antonio de Nebrija, 1, 1998, p. 143-209

MARTÍNEZ NEIRA, Manuel: El estudio del derecho. Libros de texto y planes de estudio en la Universidad contemporánea. Madrid: Instituto Antonio de Nebrija de estudios sobre la Universidad, Universidad Carlos III, Dykinson, 2001 (Biblioteca del Instituto Antonio de Nebrija de estudios sobre la Universidad, 4)

MEDINA ENCINA, Matilde: "Criterios de organización de un centro de documentación jurídica", en Documentación de las ciencias de la información, $\mathrm{n}^{\mathrm{o}}$ 17, 1994, pp. 241-247 
MIGUEL ALONSO, Aurora: "Del Plan Pidal a la Ley Moyano: consolidación de la Biblioteca de la Universidad Central", en Estudios históricos. Homenaje a los profesores José $M^{a}$ Jover Zamora y Vicente Palacio Atard. Madrid: UCM, Departamento de Historia Contemporánea, 1990, pp. 681-701

MIGUEL ALONSO, Aurora: "Los estudios de doctorado y el inicio de la tesis doctoral en España. 1847-1900". Separata de Archivos universitarios e Historia de las Universidades, 9, 2003, pp.197-222

MOREAU, Marie Christine: "La prensa digital española: la configuración de un nuevo medio", en Prensa, impresos, lectura en el mundo hispánico contemporáneo. Homenaje a Jean- François Botrel. Edición a cargo de Jean-Michel Desvois. Pessac Cedex: PILAR (Prensa, Impresos, Lectura en el Área Románica), 2005, pp.572-584

PAEZ MAÑÁ, Jorge: Bases de datos jurídicos. Madrid: CINDOC, 1994

PÉREZ-PRENDES MUÑOZ-ARRACO, José Mª : Crónica histórica de la Facultad de Derecho. Madrid: Universidad Complutense de Madrid, Facultad de Derecho, 2000

RIAZA MARTÍNEZ-OSORIO, Román: Historia de la literatura jurídica española. Notas de un curso, por-----Profesor Auxiliar de la asignatura en la Universidad Central, y Catedrático, excedente, de Historia del Derecho Español. Madrid: Litografía E. Nieto, 1930

RIAZA MARTÍNEZ-OSORIO, Román: Historia de la literatura jurídica española. Madrid: Universidad Complutense, 1998

RUS RUFINO, Salvador: "La enseñanza del derecho natural en el último tercio del siglo XIX", en Manuales y textos de enseñanza en la universidad liberal. VII Congreso internacional sobre la historia de las universidades hispánicas. Madrid: Instituto Antonio de Lebrija de estudios sobre la Universidad, universidad Carlos III de Madrid, 2004, pp.655-679

SÁNCHEZ DE CASTRO, FRANCISCO: Apuntes de literatura y bibliografía jurídicas de España. Madrid: Imprenta de los señores Lezcano y Co., 1883

SÁNCHEZ ILLÁN, Juan Carlos: "La edición de periódicos y la empresa periodística", en Historia de la edición en España (1836-1936). Dirigido por Jesús A. Martínez Martín. Madrid: Marcial Pons, 2001, pp.397-414

TORRES CAMPOS, Manuel: Estudios de Bibliografía española y extranjera del Derecho y del Notariado. Madrid: Academia Matritense del Notariado, Establecimiento Tipográfico de Eduardo Cuesta, 1878

TORRES CAMPOS, Manuel: Nociones de Bibliografia y Literatura jurídicas de España. Madrid: Góngora, 1884

TORRES CAMPOS, Manuel: Bibliografia española contemporánea del Derecho y de la Politica. 1800-1880: con tres apéndices relativos a la bibliografia extranjera sobre el derecho español, a la hispanoamericana y a la portuguesa-brasileña. Madrid, etc: Librería de Fernando Fe, etc, 1883-1897

VILLASEÑOR RODRÍGUEZ, Isabel: "La cátedra de bibliografía jurídica de la Universidad Central de Madrid (1883-1936)", en Revista general de información y documentación, vol. 16, $\mathrm{n}^{\mathrm{o}}$ 2, 2006, pp. 65-91 
VILLASEÑOR RODRÍGUEZ, Isabel: "Principales recursos de información para la filosofía jurídica: las guías de fuentes", en RDUNED. Revista Derecho UNED, $2008, n^{\circ} 3$, pp. 261-288

VILLASEÑOR RODRÍGUEZ, Isabel: Fuentes de información en filosofia jurídica española (siglos XIX-XXI). Madrid: Dykinson, 2009

VILLASEÑOR RODRÍGUEZ, Isabel: "Instituciones de interés para la Filosofía jurídica como fuentes de información", en Revista General de información y documentación, 2009, v. 19, pp. 297-316 